\title{
Combretazet-3 a novel synthetic cis-stable combretastatin A-4- azetidinone hybrid with enhanced stability and therapeutic efficacy in colon cancer
}

\author{
LISA M. GREENE ${ }^{1}$, SHU WANG ${ }^{2}$, NIAMH M. O'BOYLE ${ }^{2}$, SANDRA A. BRIGHT ${ }^{1}$, JANE E. REID ${ }^{1}$, \\ PATRICK KELLY ${ }^{2}$, MARY J. MEEGAN ${ }^{2}$ and DANIELA M. ZISTERER ${ }^{1}$ \\ Schools of ${ }^{1}$ Biochemistry and Immunology, ${ }^{2}$ Pharmacy and Pharmaceutical Sciences, \\ Trinity Biomedical Sciences Institute, Trinity College Dublin, Dublin 2, Ireland
}

Received September 28, 2012; Accepted December 20, 2012

DOI: $10.3892 /$ or.2013.2379

\begin{abstract}
In recent years an extensive series of synthetic combretastatin A-4 (CA-4)-azetidinone ( $\beta$-lactam) hybrids were designed and synthesised with a view to improve the stability, therapeutic efficacy and aqueous solubility of CA-4. Lead compounds containing a 3,4,5-trimethoxy aromatic ring at position 1 and a variety of substitution patterns at positions 3 and 4 of the $\beta$-lactam ring were screened in three adenocarcinoma-derived colon cancer cell lines (CT-26, Caco-2 and the CA-4 resistant cell line, HT-29). In both CT-26 and Caco-2 cells all $\beta$-lactam analogues analysed displayed potent therapeutic efficacy within the nanomolar range. Substitution of the ethylene bridge of CA- 4 with the $\beta$-lactam ring together with the aforementioned aryl substitutions improved the therapeutic efficacy of CA-4 up to 300-fold in the combretastatin refractory HT-29 cells. The lead compound combretazet-3 (CAZ-3); chemical name [4-(3-hydroxy-4-methoxyphenyl)-3(4-hydroxyphenyl)-1-(3,4,5-trimethoxyphenyl)azetidin-2-one] demonstrated improved chemical stability together with enhanced therapeutic efficacy as compared with CA-4 whilst maintaining the natural biological properties of CA-4. Furthermore, CAZ-3 demonstrated significant tumour inhibition in a murine model of colon cancer. Our results suggest that combretastatin-azetidinone hybrids represent an effective novel therapy for the treatment of combretastatin resistant carcinomas.
\end{abstract}

\section{Introduction}

Microtubules are a principle component of the cytoskeleton and play a key role in numerous biological functions including

Correspondence to: Dr Lisa M. Greene, School of Biochemistry and Immunology, Trinity Biomedical Sciences Institute, Trinity College Dublin, 152-160 Pearse Street, Dublin 2, Ireland

E-mail: greeneli@tcd.ie

Key words: combretastatin A-4, azetidinone ( $\beta$-lactam), colon cancer cell division and organelle transport. The pivotal role of tubulin in both the formation of the mitotic spindle and chromosomal separation promoted the surge in the development of both natural and synthetic microtubule targeting agents (MTAs). One such naturally occurring drug, combretastatin A-4 (CA-4; Fig. 1) was originally described by Pettit et al (1). The structure of CA-4 proved readily amenable to chemical manipulation to improve the stability, solubility and therapeutic index of this class of MTAs. Over the past two decades a vast array of synthetic CA-4 analogues were designed and synthesised with many surpassing the stability, solubility and therapeutic efficacy of the parent compound (2). The clinical success of the synthetic prodrug of CA-4, combretastatin-A4 phosphate (CA-4P) in the treatment of anaplastic thyroid carcinoma (www.clinicaltrials.gov) (3) has maintained an active interest in the chemical manipulation of CA-4 with the view to further enhancing the therapeutic efficacy of this lead compound. Furthermore, amino acid containing prodrugs of CA-4 are also undergoing clinical evaluation including AVE8062 (www. clinicaltrials.gov) (4). Structural modifications of CA-4 can be divided into three areas, those involving the manipulation of either ring $\mathrm{A}$, ring $\mathrm{B}$ or those involving the substitution of the double bond (ethylene bridge structure) connecting both rings (Fig. 1). These A and B substituted aromatic rings fit into the $A$ and $B$ pockets of the colchicine binding site on tubulin. Data collated from numerous structural activity relationship (SAR) studies confirm that a non-planar cis conformation is essential for the tubulin binding properties of CA-4. Furthermore, the majority of studies suggest that the 3,4,5-trimethoxy-substituted aromatic A-ring should be conserved to maintain maximum anticancer activity. However, contrary to this, a recent study conducted by Beale et al (5) showed that substitution of the larger meta-methoxy groups of triazole CA-4 derivatives with smaller halogen atoms yielded more potent CA-4/CA-1 analogues. Several independent studies have demonstrated that the therapeutic activity of CA-4 can also be enhanced by the strategic modification of ring B (6). Apart from strategies to improve the potency of CA-4 other main areas of research focused on methods to overcome the solubility issues of CA-4 and also to prevent the undesired conversion into the inactive trans isomer (Fig. 1). Modification of the phenolic group on 
ring B forming either a phosphate or an amino acid ester was demonstrated to be an effective method of improving the solubility of CA-4 whilst retaining optimum biological activity. Double bond isomerisation can be prevented by the strategic inclusion of various types of heterocyclic rings in place of the usual ethylene bridge structure of CA-4 (7).

In recent years our group has designed and synthesised an extensive series of azetidinone ( $\beta$-lactam) CA- 4 analogues with a view to overcoming double bond isomerisation by substituting the ethylene bridge structure for a 1,4-diaryl2 -azetidinone ring. The rigid $\beta$-lactam ring scaffold allows a similar spatial arrangement between the two aromatic rings as observed in the non-planar cis-conformation of CA-4 while permanently preventing the undesired conversion to the inactive trans-configuration (8). Further studies demonstrated that the inclusion of an aromatic ring at position 3 of the $\beta$-lactam significantly improved the potency of the series. Hence, a $\beta$-lactam substituted at position 3 with a phenolic ring soon became the core structure for future designs (9). Solubility issues of the CA-4-azetidinone analogues were addressed by esterification of the $3 \mathrm{OH}$ group of ring $\mathrm{B}$ with phosphates and amino acids (unpublished data).

However, despite the significant advances made in recent years in terms of improving the solubility and stability of CA-4, the lack of therapeutic efficacy as a single agent and the emergence of resistance to CA-4 has somewhat hindered the clinical and commercial success of this compound. We recently reported that CA-432, a lead combretastatin-azetidinone hybrid was 10 -fold more potent than CA-4 in CA-4 refractory HT-29 cells, suggesting a possible functional advantage of the ethylene bridge-azetidinone substitution (10). In this study, we screened selected combretastatin-azetidinone hybrids (hereafter referred to as combretazets) to further characterise the structure activity relationship of these compounds in the CA-4 resistant HT-29 cells. The stability and therapeutic potential of a lead compound combretazet-3 (CAZ-3) as a single agent in the murine CT-26 colon cancer model was evaluated.

\section{Materials and methods}

Compounds. CA-4 and bafilomycin A1 were purchased from Sigma-Aldrich (Poole, Dorset, UK). 1,4-Diaryl-2-azetidinone analogues were synthesised as previously described by Carr et al (8) CAZ-1, CAZ-2 and CAZ-3 (9), CAZ-4 (7), CAZ-5 (11), CAZ-2P, CAZ-3P, CAZ-4P, CAZ-6 and CAZ-7 (unpublished data). All general reagents unless stated otherwise were purchased from Sigma. Bafilomycin A1 was dissolved in DMSO. CA-4 and all analogues were prepared as a $10-\mathrm{mM}$ stock in ethanol and stored at $-20^{\circ} \mathrm{C}$.

Cell culture. CT-26 cells are a chemically (N-nitroso-Nmethylurethane) induced, undifferentiated murine colon carcinoma fibroblast cell line originating from $\mathrm{BALB} / \mathrm{c}$ mice. HT-29 and Caco-2 cells originate from a human adenocarcinoma of the colon and were originally obtained from the European Collection of Cell Cultures. All cells were grown in DMEM Glutamax media. CT-26 and HT-29 media were supplemented with $10 \%$ foetal bovine serum (FBS) and Caco-2 were cultivated with $20 \%$ FBS. Both CT-26 and Caco-2 media were supplemented with $1 \%$ non-essential amino acids (NEAA). All media contained $100 \mathrm{U} / \mathrm{ml}$ penicillin and $100 \mu \mathrm{g} / \mathrm{ml}$ streptomycin. Cells were maintained at $37^{\circ} \mathrm{C}$ in $5 \% \mathrm{CO}_{2}$ in a humidified incubator. Cell culture materials were supplied from Gibco, Invitrogen Corp. (Grand Island, NY, USA). All cells were sub-cultured 3 times/week by trypsinisation.

Alamar blue cell viability assay. Cell proliferation was analysed using the Alamar Blue assay (Invitrogen Corp.) according to the manufacturer's instructions. Cells were seeded at a density of $5 \times 10^{3}$ cells/well (CT-26) or $1 \times 10^{4}$ cells/well (Caco-2, HT-29) in triplicate in 96-well plates. After $24 \mathrm{~h}$, cells were then treated with either medium alone, vehicle [1\% ethanol (v/v)] or with serial dilutions of CA-4 or combretazets. After $72 \mathrm{~h}$, Alamar Blue $[10 \%(\mathrm{v} / \mathrm{v})]$ was added to each well and plates were incubated for $3-5 \mathrm{~h}$ at $37^{\circ} \mathrm{C}$ in the dark. Fluorescence was read using a 96-well fluorimeter with excitation at $530 \mathrm{~nm}$ and emission at $590 \mathrm{~nm}$. Results were expressed as percentage viability relative to vehicle control (100\%). Dose response curves were plotted and $\mathrm{IC}_{50}$ values (concentration of drug resulting in 50\% reduction in cell survival) were obtained using the commercial software package Prism (GraphPad Software, Inc., La Jolla, CA, USA). Experiments were performed in triplicate on at least three separate occasions.

Cell cycle detection. After treatment, cells were collected then centrifuged at $800 \mathrm{x}$ g for 10 min and fixed with $70 \%$ ethanol overnight at $-20^{\circ} \mathrm{C}$. The ethanol was removed by centrifugation at $800 \mathrm{x} \mathrm{g}$ for $10 \mathrm{~min}$. The cells were then stained in PBS containing $0.5 \mathrm{mg} / \mathrm{ml} \mathrm{RNase} \mathrm{A} \mathrm{and} 0.15 \mathrm{mg} / \mathrm{ml}$ propidium iodide and then incubated for $30 \mathrm{~min}$ in the dark at $37^{\circ} \mathrm{C}$. Cell cycle distribution was analysed by flow cytometry at $488 \mathrm{~nm}$ using the FACSCalibur flow cytometer (Becton-Dickinson, San Jose, CA). All data were recorded and analysed using the CellQuest Software (Becton-Dickinson).

Plasma and pH stability studies. Peripheral blood was collected from healthy donors with informed consent and was made anonymous prior to use. The plasma was separated by Ficoll-gradient and diluted (1:9) with PBS pH 7.4 and warmed to $37^{\circ} \mathrm{C}$. The $\mathrm{pH}$ stability study was carried out in PBS pH 3.0. Test compounds $(1.5 \mathrm{mg} / \mathrm{ml})$ were dissolved in acetonitrile at time $\mathrm{t}=0$. Plasma solution containing test compound $(250 \mu \mathrm{l})$ was added to $2 \%(\mathrm{w} / \mathrm{v}) \mathrm{ZnSO}_{4}$ solution in acetonitrile: water (1:1) $(500 \mu \mathrm{l})$. Aliquots were taken at the specified time intervals, vortexed, centrifuged for $3 \mathrm{~min}$ at 9,500 $\mathrm{x}$ g before injection onto an HPLC column (Varian Pursuit XRs C18 reverse phase 250x4.6 mm chromatography column) to determine the stability using a Waters 2487 Dual Wavelength Absorbance Detector, a Waters 1525 Binary HPLC Pump, a Waters In-Line Degasser AF and a Waters 717 plus Autosampler. Samples were detected using a wavelength of $254 \mathrm{~nm}$. All samples were analysed using acetonitrile (60\%):water $(40 \%)$ with $0.1 \%(\mathrm{v} / \mathrm{v})$ trifluoroacetic acid over $10 \mathrm{~min}$ and a flow rate of $1 \mathrm{ml} / \mathrm{min}$ to evaluate the percentage decline in the predetermined peak area for each compound. The retention times for CA- 4 and CAZ-3 were 5.9 and $3.7 \mathrm{~min}$, respectively. The percentage recovery was calculated using the following formula [(plasma or $\mathrm{pH} 3.0$ peak area/mean aqueous peak area) x 100]. 

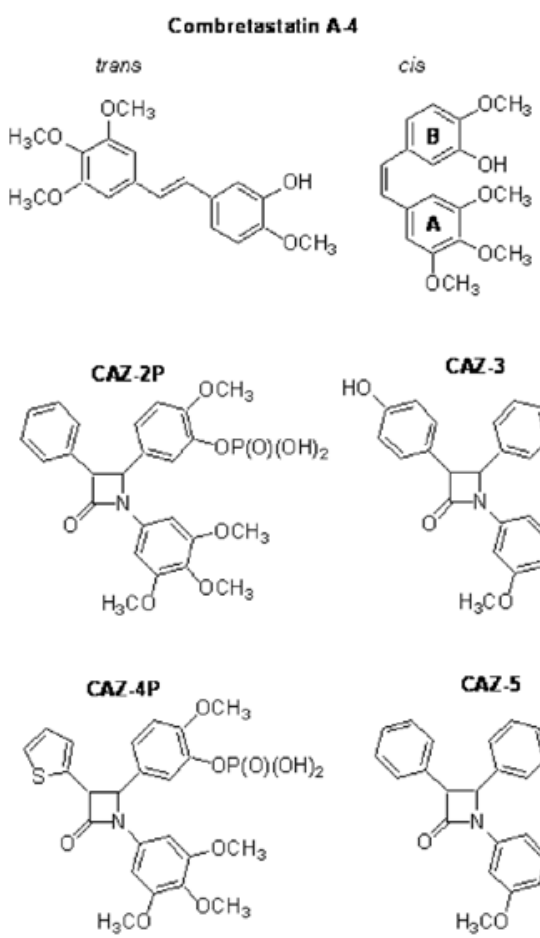
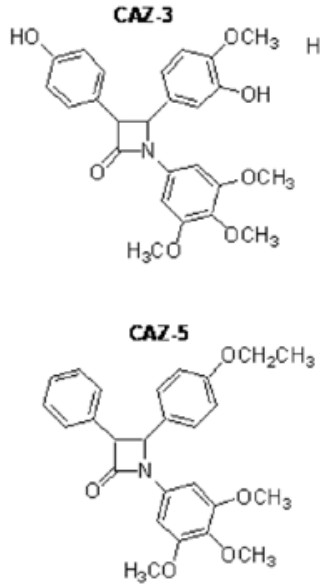
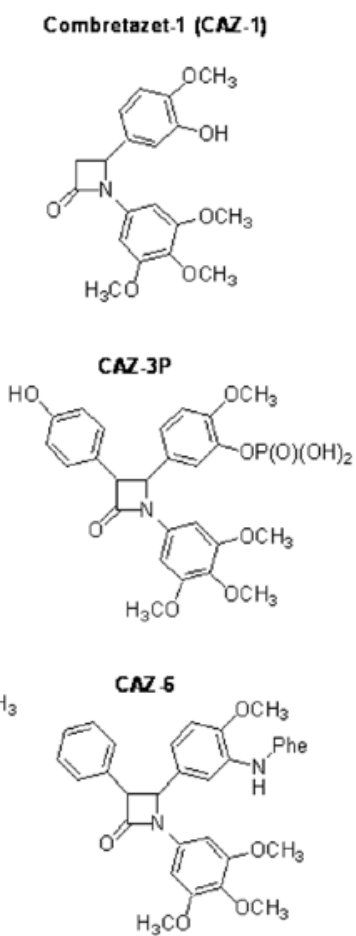

CAZ.2

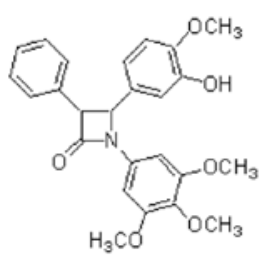

Figure 1. Structures and nomenclature of combretastatin-A4 and selected combretazets.

Human microsomal stability study. Microsomal stability was determined using pooled human liver microsomes (The UK Human Tissue Bank, Leicester, UK). Ethical approval was obtained from south Cheshire Local Research Ethics Committee (Chester, UK). Test compound (3 $\mu \mathrm{M})$ together with microsome protein $(0.5 \mathrm{mg} / \mathrm{ml}), 1 \mathrm{mM}$ NADPH in $0.1 \mathrm{M}$ phosphate buffer $\mathrm{pH} 7.4$ was incubated for $0,5,15,30$ and 45 min. The negative control did not contain NADPH. The samples were quenched with methanol and the protein was precipitated by centrifugation for $20 \mathrm{~min}$ at $1,100 \mathrm{x} \mathrm{g}$ at $4^{\circ} \mathrm{C}$. Supernatants were then analysed by LC/MS. The In peak area ratio (compound peak area/internal standard peak area) was plotted against time and the slope of the line determined to give the elimination rate constant $[\mathrm{K}=(-1)$ (slope)]. The half life $\left(t_{1 / 2}\right)$ and the in vitro intrinsic clearance $\left(\mathrm{CL}_{\mathrm{int}}\right.$ $\mu \mathrm{l} / \mathrm{min} / \mathrm{mg}$ protein) were calculated by the following equations; $t_{1 / 2}=0.693 / \mathrm{K} ; \mathrm{CL}_{\text {int }}=\mathrm{V}(0.693) / t_{1 / 2}$ where $\mathrm{V}$, incubation volume in $\mu \mathrm{l} / \mathrm{mg}$ microsomal protein.

Quantification of AVOs with acridine orange staining using flow cytometry. Autophagy is characterised by the formation and promotion of acidic vesicular organelles (AVOs). The formation of acidic compartments was quantified by flow cytometric analysis of acridine orange stained cells (10). Acridine orange stains the cytoplasm green and the nucleus a dim red, whereas acidic compartments fluoresce bright red. The intensity of the red fluorescence is proportional to the amount of acidity. Following treatment, cells were stained with acridine orange $1 \mu \mathrm{g} / \mathrm{ml}$ for $15 \mathrm{~min}$ at $37^{\circ} \mathrm{C}$. Bafilomycin A1 (5 nM) was dissolved in DMSO and added to the cells $45 \mathrm{~min}$ prior to the addition of acridine orange. Cells were then trypsinised and collected in phenol-red free medium. Green (510-530 nm) and red $(650 \mathrm{~nm})$ fluorescence emission from $10^{4}$ cells illuminated with blue (488 nm) excitation light was measured with a CyAn ADP Flow Cytometry Analyzer (Beckman Coulter, Nyon, Switzerland). The red:green fluorescence ratio for individual cells was calculated using FlowJo software (Tree Star, Inc., San Carlos, CA).

In vivo studies. Tumour growth was initiated by subcutaneous injection of a CT-26 cell suspension $\left(10^{6}\right.$ cells) into the right flank of 6-8 week old female Balb/c mice. The experiments were conducted on Day 7 when tumours reached a maximum diameter range of 3.6-6.3 mm. Mice were randomly divided into two groups of five. The treatment group received a single intraperitoneal (i.p.) injection of $40 \mathrm{mg} / \mathrm{kg} \mathrm{CAZ-3} \mathrm{dissolved} \mathrm{in}$ ethanol:cremophore:PBS [10\%:10\%:80\% (v/v)] and the control group received one i.p. injection of vehicle only. Tumour growth was measured every second day with a sterile vernier callipers. The long (L) and short $(\mathrm{S})$ axes were recorded and tumour volume $(\mathrm{V})$ was calculated using the following equation $\mathrm{V}=\left(\mathrm{S}^{2} \mathrm{xL}\right) / 2$. Mice were culled by $\mathrm{CO}_{2}$ asphyxiation at the experimental end point. Ethical approval was obtained from the Research Ethical Approval Committee, Trinity College Dublin. The study was performed under the license number: B100/4275 granted by Department of Health and Children, Hawkins House, Dublin 2, Ireland.

\section{Results}

The effects of CA-4 and selected combretazets on the viability of colon cancer-derived cell lines. The synthetic combretazets were designed and synthesised with a view to improve the stability, therapeutic efficacy and aqueous solubility of the parent compound CA-4, hence many in the series contained a phenolic group, phosphate ester or an amino group (Fig. 1). 
Table I. Evaluation of CA-4 and selected combretazets in adenocarcinoma-derived colon cancer cells.

\begin{tabular}{|c|c|c|c|c|c|c|}
\hline \multirow[b]{2}{*}{ Compound } & \multicolumn{2}{|c|}{ CT-26 } & \multicolumn{2}{|c|}{ Caco-2 } & \multicolumn{2}{|c|}{ HT-29 } \\
\hline & $\mathrm{IC}_{50}(\mathrm{nM})$ & RI & $\mathrm{IC}_{50}(\mathrm{nM})$ & RI & $\mathrm{IC}_{50}(\mathrm{nM})$ & RI \\
\hline CA-4 & 5.7 & 1.0 & 41.2 & 1.0 & 9020 & 1.0 \\
\hline CAZ-1 & 11.8 & -2.1 & 236.0 & -5.7 & 1011 & +8.1 \\
\hline CAZ-2 & 13.46 & -2.4 & 113.6 & -2.8 & 909 & $+9(11)$ \\
\hline CAZ-3 & 4.25 & +1.4 & 15.3 & +5.6 & 50 & +180.0 \\
\hline CAZ-4 & 165.0 & -28.9 & 28.3 & +1.5 & 3510 & +2.6 \\
\hline CAZ-5 & 136.9 & -24.1 & ND & ND & 30 & +300.0 \\
\hline CAZ-6 & 118.0 & -20.7 & 120.7 & -2.9 & 70 & +128.9 \\
\hline CAZ-7 & 245.3 & -43.0 & 704.2 & -17.1 & 260 & +34.7 \\
\hline CAZ-2P & 75.17 & -13.2 & 96.7 & -2.4 & 440 & +20.5 \\
\hline CAZ-3P & 8.0 & -1.4 & 109.3 & -2.7 & 119 & +7.6 \\
\hline CAZ-4P & 9.7 & -1.7 & ND & ND & 4670 & +1.93 \\
\hline
\end{tabular}

Cells were exposed to multiple concentrations of the indicated compound for $72 \mathrm{~h}$. Cell viability was assessed using the Alamar Blue assay and respective $\mathrm{IC}_{50}$ values were calculated. ND, not determined. RI, relative inhibition, compared to CA-4. The most active analogue, CAZ-3, is highlighted in bold.

Table II. Calculation of intrinsic clearance values $\left(\mathrm{CL}_{\mathrm{int}}\right)$ for CA-4 and CAZ-3 in human microsomes.

\begin{tabular}{lcc}
\hline Compound & $\mathrm{CL}_{\text {int }}(\mu \mathrm{l} / \mathrm{min} / \mathrm{mg}$ protein $)$ & $t_{1 / 2}(\mathrm{~min})$ \\
\hline CA-4 & $157.0 \pm 18.9$ & 8.83 \\
CAZ-3 & $76.7 \pm 21.4$ & 18.1 \\
\hline
\end{tabular}

Compounds $(3 \mu \mathrm{M})$ were incubated in the presence of NADPH $(1 \mathrm{mM})$ and human liver microsomes $(0.5 \mathrm{mg} / \mathrm{ml})$ and intrinsic clearance values were calculated as described in Materials and methods. Clearance values are expressed as $(\mu 1 / \mathrm{min} / \mathrm{mg})$ of microsomal protein. Values represent the mean \pm SEM of at least triplicate determinations.

All combretazets analysed were effective in the nanomolar range in drug-sensitive CT-26 and Caco- 2 cells and were more potent than CA-4 in CA-4 refractory HT-29 cells (Table I). Compound CAZ-2 is identical to CA-4 with the exception of the azetidinone-ethylene bridge substitution and demonstrated an 8-fold increase in activity in HT-29 cells confirming a functional advantage of the ethylene bridge-azetidinone substitution in overcoming combretastatin resistance. Compounds containing a B-ring meta-hydroxy group (CAZ-1, CAZ-2 and CAZ-4) or a phosphate (CAZ-2P, CAZ-3P and CAZ-4P) conjugate were the least active of the series in the combretastatin refractory HT-29 cells. Deletion (CAZ-5) or substitution of the B-ring meta-hydroxy group with an amine conjugated amino acid (CAZ-6 and CAZ-7) significantly increased activity of the series with $\mathrm{IC}_{50}$ values in the nanomolar range in combretastatin refractory HT-29 cells. However, CAZ-3 with a B-ring meta-hydroxy group was the exception to this observation. CAZ-3 was more potent than CA-4 in all three adenocarcinoma-derived cell lines tested. Hence, CAZ-3 was selected for further biological analysis.
Table III. Suppression of tumour growth by CAZ-3.

\begin{tabular}{lccc}
\hline Group & $\begin{array}{c}\text { Tumour volume } \\
\text { (Day 7) }\end{array}$ & $\begin{array}{c}\text { Tumour volume } \\
\text { (Day 11) }\end{array}$ & Mortality \\
\hline Control & $37.08 \pm 8.326$ & $263.2 \pm 62.13$ & $0 / 5$ \\
CAZ-3 & $42.45 \pm 11.45$ & $77.62 \pm 19.24$ & $1 / 5$ \\
Single IP & $\mathrm{NS}$ & $<0.05^{\mathrm{a}}$ & \\
$40 \mathrm{mg} / \mathrm{kg}$ & & & \\
\hline
\end{tabular}

BALB/c mice $(n=10)$ were inoculated subcutaneously with $10^{6} \mathrm{CT}-26$ cells. On Day 7 mice received a single i.p. injection of vehicle (ethanol:cremophore:PBS; 10\%:10\%:80\%) or $40 \mathrm{mg} / \mathrm{kg}$ CAZ-3. Tumour growth was measured every second day using a sterile vernier callipers. Values represent the mean \pm SEM (Student's t-test; ${ }^{\mathrm{a}} \mathrm{P}<0.05$; NS, not significant).

The effects of CA-4 and selected combretazets on the cell cycle in CT-26 cells. CT-26 cells were selected for further analysis given that all combretazets were effective in the nanomolar range in this cell line. The effects of CA-4, CAZ-3 and its corresponding prodrug CAZ-3P on the cell cycle and cell death were assessed by flow cytometric analysis of propidium iodide stained CT-26 cells. The percentage of cell death was estimated by the quantification of the pre- $G_{1}$ peak. As shown in Fig. 2, all compounds tested produced an early $\mathrm{G}_{2} \mathrm{M}$ cell cycle arrest at $8 \mathrm{~h}$ followed by a significant time-dependent increase in cell death.

Induction of autophagy by CAZ-3. Our recent findings demonstrated that CA-4 and CAZ-2 (CA-432) induced autophagy in adenocarcinoma-derived colon cells as confirmed by acridine orange staining of vesicle formation, electron microscopy and increased expression of LC3-II (10). Hence, the effect of 

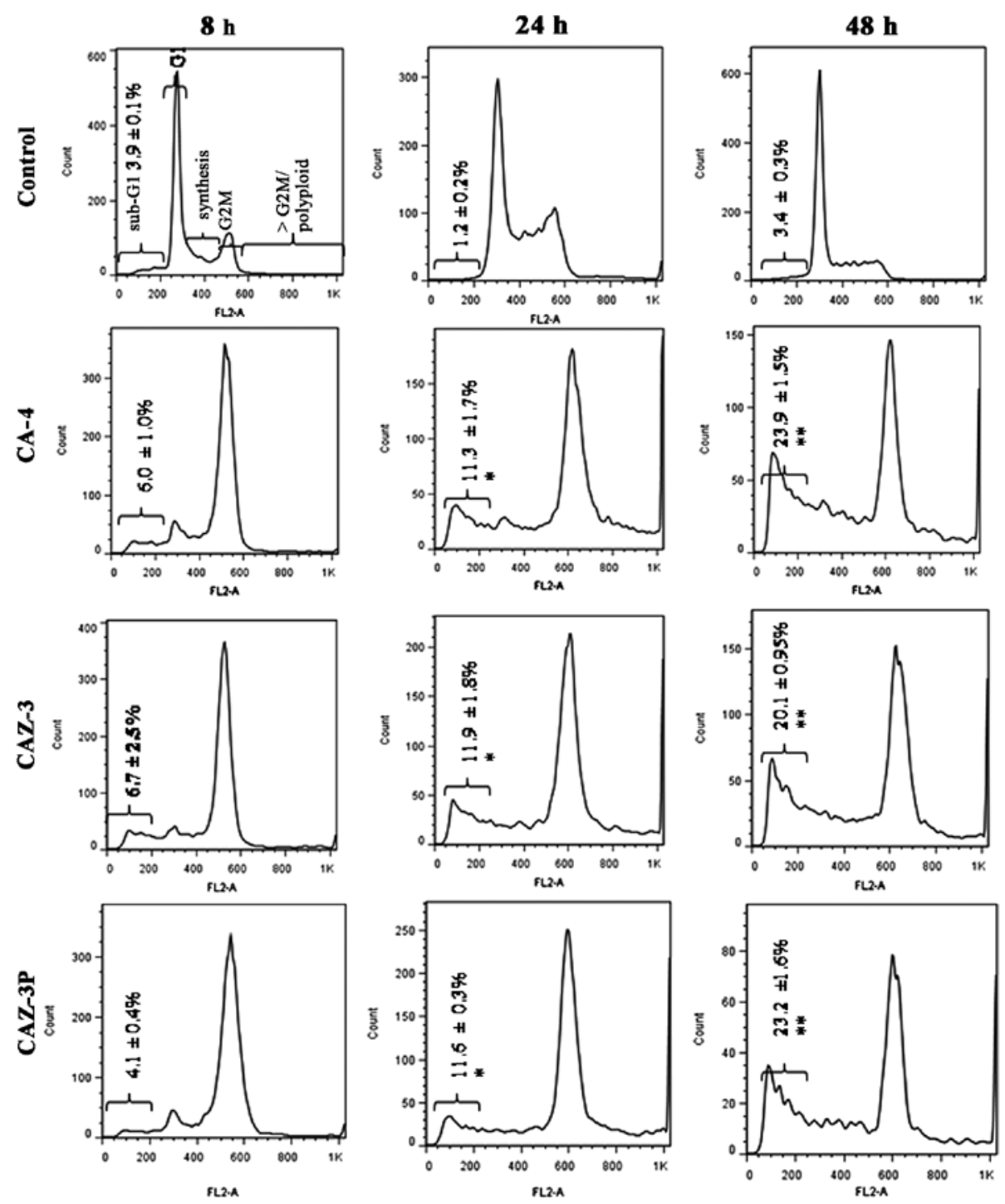

Figure 2. CAZ-3 and its prodrug CAZ-3P induce $\mathrm{G}_{2} \mathrm{M}$ cell cycle arrest and cell death in a similar manner to CA-4 in murine CT-26 adenocarcinoma cells. CT-26 cells were treated with vehicle [ethanol $0.2 \%(\mathrm{v} / \mathrm{v})$ ] or $50 \mathrm{nM}$ of compound for the times indicated. Cells were then fixed, stained with PI and analysed by flow cytometry. The percentage of cell death was determined by quantification of the sub- $\mathrm{G}_{1}$ peak. Values represent the mean $\pm \mathrm{SEM}$ for three independent experiments (Student's t-test: ${ }^{*} \mathrm{P}<0.05,{ }^{* *} \mathrm{P}<0.01$ ).

CAZ-3 on autophagic vesicle formation was evaluated by flow cytometric analysis of acridine orange stained cells. Fig. 3 indicates that like other combretastatins CAZ-3 also induced autophagy in adenocarcinoma cells. Furthermore, CAZ-3 also induced autophagy in HT-29 and Caco-2 adenocarcinomaderived colon cancer cells (data not shown). Numerous studies have demonstrated a dependence of the acidification of cellular organelles on the vacuolar $\mathrm{H}^{+}$ATPase using the specific inhibitor bafilomycin A1. Similarly, pretreatment of CT-26 cells with bafilomycin A1 significantly inhibited CAZ-3 induced autophagy (Fig. 3).

CAZ-3 is more stable than CA-4 in both human plasma and microsomes. The stability of CA-4 and CAZ-3 in acidic media and in human plasma was next determined by HPLC. Both compounds were stable in plasma at physiological $\mathrm{pH} 7.3$ for up to $5 \mathrm{~h}$ (Fig. 4A) and remained stable up to $24 \mathrm{~h}$ (data not shown). Under acidic conditions ( $\mathrm{pH}$ 3.0) CAZ-3 is more stable than CA-4 (Fig. 4B). These findings are in agreement with other studies demonstrating instability of CA-4 in acidic media (12). Microsome stability was determined using pooled human liver microsomes (Table II). The $\beta$-lactam bridge improved the metabolic stability of CAZ-3 as compared with CA-4 by doubling the in vitro clearance time.

CAZ-3 significantly inhibited the growth of CT-26 cells grafted to mice. To study the effects of CAZ-3 on tumour growth we selected the CT-26 murine model of colon carcinoma, a model frequently used to test the efficacy of CA-4 and its synthetic analogues (13). Furthermore, experimental models involving xenografts of human tumours in a mouse host may lack some of the critical tumour host interactions. In the antitumour efficacy experiment mice received a single IP injection of $40 \mathrm{mg} / \mathrm{kg}$ on Day 7 when tumours were on average $5 \mathrm{~mm}$ in diameter. On Day 7 there was no significant difference between control and CAZ-3 treated groups. By Days 9 (data not shown) and 
A
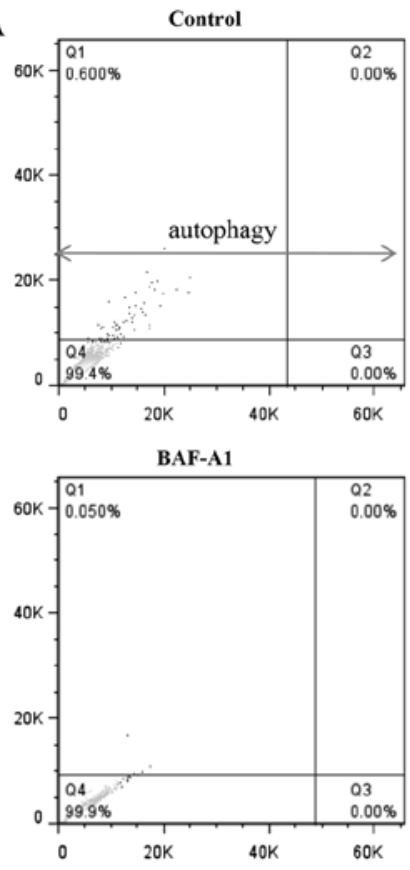
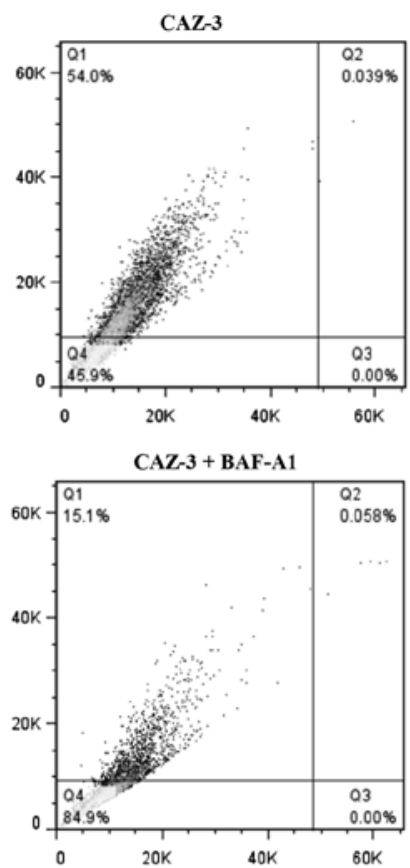

B

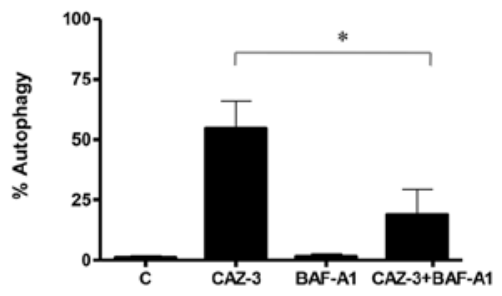

Figure 3. CAZ-3 induces autophagy in adenocarcinoma-derived cell lines. Cells were treated with ethanol vehicle [0.2\% (v/v)] or $50 \mathrm{nM}$ CAZ-3 \pm BAF-A1 $(5 \mathrm{nM})$. BAF-A1 was added $45 \mathrm{~min}$ before the addition of acridine orange. After $48 \mathrm{~h}$, cells were stained with $1 \mu \mathrm{g} / \mathrm{ml}$ acridine orange for $15 \mathrm{~min}$. The differential staining of acridine orange can detect the acidic vesicles of autophagic cells. (A) Representative flow cytometry dot plots are shown of acridine orange stained CT-26 cells. The acidic vesicles of the autophagic cells (top left and right quadrants) were quantified by FlowJo software. (B) Values represent the mean \pm SEM for at least three independent experiments (Student's t-test: ${ }^{*} \mathrm{P}<0.05$ ).
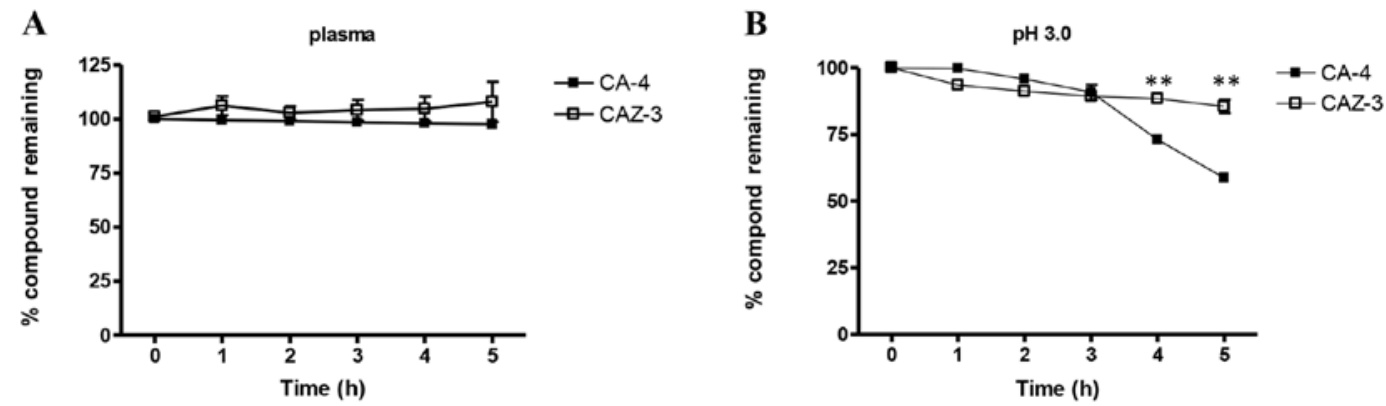

Figure 4. Analysis of the stability of CA-4 and CAZ-3 in plasma and at pH 3.0 over time. Compounds were incubated at $37^{\circ} \mathrm{C}$ in plasma pH 7.3 (A) or $\mathrm{PBS}$ $\mathrm{pH} 3.0$ (B) and aliquots were taken and analysed by HPLC at various time intervals. The percentage recovery was calculated using the formula [(plasma or pH 3.0 peak area/mean aqueous peak height) x 100]. The absence of an error bar indicates that the error was smaller than the size of the symbol. Results are expressed as the mean \pm SEM for three independent experiments (two-way ANOVA with Bonferroni post tests: ** $\mathrm{P}<0.001$ ).

11 (Table III), CAZ-3 significantly inhibited tumour growth. Both a rough coat and diarrhoea were observed in $100 \%$ of CAZ-3 treated mice.

\section{Discussion}

Several water soluble CA-4 analogues including CA-4P (ZYBRESTAT), CA-1P (OXi4503) and AC7700 (AVE 8062) are currently undergoing clinical trials as vascular targeting agents (www.clinicaltrials.gov) $(14,15)$. However, these compounds all contain the isomerisable olefinic bond which may hinder the continued clinical success of the compounds. To date there is no cis-stable CA-4 analogue undergoing clinical trials and hence there is a demand for pre-clinical data on potent $c i s$-restricted CA-4 analogues. The combretazets are a novel class of synthetic combretastatin and azetidinone ( $\beta$-lactam) hybrids that function through a combretastatin-like mechanism. Overall the combretastatins and the combretazets are structurally and functionally similar. Both classes exhibit a similar spatial arrangement between the two phenyl A and B rings but differ in the bridge structure connecting the rings. The strategic ethylene bridge-azetidinone substitution produced cis-stable analogues with improved chemical stability and ease of synthesis. Extensive biochemical analysis demonstrated that the ethylene bridge-azetidinone substitution did not influence the biological properties of CA-4 $(9,10,16,17)$. In more detail, both classes of drugs inhibit the polymerisation of tubulin inducing a range of cellular responses including; $\mathrm{G}_{2} \mathrm{M}$ cell cycle arrest, autophagy, mitotic catastrophe, caspase-dependent cell death and caspase-independent cell death. In this report, we 
demonstrate that further substitutions to the aromatic ring at position 3 of the azetidinone yielded a superior compound with enhanced stability and potency against combretastatin refactory adenocarcinoma-derived cells and tumours without altering the biological properties of CA-4. The lead compound CAZ-3 inhibited the polymerisation of tubulin (9), induced a $\mathrm{G}_{2} \mathrm{M}$ cell cycle arrest and a time-dependent increase in cell death $\left(\mathrm{sub}-\mathrm{G}_{1}\right.$ ) in the colon adenocarcinoma-derived CT-26 cells in a similar manner to its phosphate prodrug counterpart (CAZ-3P) and CA-4. As recently observed with CA-4 (10), CAZ-3 also induced autophagy in CT-26 adenocarcinoma cells. Autophagy is a highly regulated self-catabolic process which can facilitate a prolonged cell survival in spite of adverse stress by generating energy via lysosomal degradation of cytoplasmic constituents (18). Furthermore, we have previously demonstrated that manipulation of autophagy can enhance the therapeutic potential of CAZ-2 (10).

The adenocarcinoma-derived HT-29 cells are inherently resistant to CA-4. The mechanism of innate resistance remains undefined. Recent studies rule out multidrug resistance protein-1 (MRP-1) mediated resistance to CA-4 in HT-29 cells (12). MRP-1 is a member of the ATP-binding cassette family of polytopic membrane transporters and is responsible for conferring resistance to a broad range of chemotherapeutic drugs (19). However, the authors demonstrate a role for MRP-1 in mediating resistance to some oxazole CA-4 derivatives (12). Data obtained from SARs from numerous independent studies on CA-4 analogues provide some insight into the possibility of structural modification of CA-4 as a means of overcoming CA-4 resistance. In this report we demonstrate that a substitution of the ethylene bridge with a $\beta$-lactam ring (CAZ-1) increased activity in HT-29 cells compared to CA-4 by 8 -fold. This finding would suggest that cis-trans isomerisation is not solely responsible for CA-4 resistance in HT-29 cells but may contribute in part to CA-4 resistance in these cells. This mechanism of resistance may be overcome by synthetic analogues featuring an ethylene bridge substitution with various types of heterocyclic rings yielding stable analogues which do not isomerise. We also report that deletion or substitution of the B-ring meta-hydroxy group with an amine conjugated amino acid in conjunction with introduction of a 3-position aromatic ring significantly enhances the activity of the series by up to 300 -fold in HT-29 cells as compared to CA-4. These findings are in agreement with a recent report by Schobert et al (12) demonstrating improved activity of oxazole bridged CA-4 analogues by substitution of the B-ring phenolic group with $\mathrm{H}$, fluoro or amino groups. Also, substitution of the ethylene bridge with a sulfone group coupled with the substitution of B-ring with a 5-amino-6-methoxyquinoline moiety yielded a novel compound with activity in the low nanomolar range in HT-29 cells $\left(\mathrm{IC}_{50}=16 \mathrm{nM}\right)$ (20). Ring B 4-ethoxyphenyl 1,5-diaryl substituted 1,2,3,4-tetrazoles also displayed potent activity in HT-29 cells (21). Taken together these findings highlight the potential of B-ring meta-hydroxy group substitutions or deletions in cis-stable analogues of CA-4 as a means of overcoming innate resistance to CA-4. However, our lead compound CAZ-3, a cis-restricted CA-4 analogue with a B-ring meta-hydroxy group demonstrated potent nanomolar activity in HT-29 cells. Molecular modelling studies highlighted a novel site of interaction between the para-phenolic 3-position of compound CAZ-3 with the colchicine binding site of tubulin (9). This unique tubulin binding characteristic is shared with compound CAZ-5 via the B-ring ethoxy group but not with CA-4 and the other listed $\beta$-lactams (11). The additional hydrophobic contact of CAZ-3 and CAZ-5 with tubulin may facilitate the observed potent antiproliferative effects observed in the CA-4 resistant HT-29 cells and offer a novel means of overcoming CA-4 resistance.

Based on promising in vitro data we proceeded to evaluate the therapeutic efficacy of CAZ-3 in the mouse CT-26 model of colon adenocarcinoma. As a single agent CA-4 failed to reduce the growth of CT-26 tumours in vivo (13). Here we report that a single injection of CAZ-3 (40 mg/kg) significantly inhibited the growth of CT-26 tumours in vivo. Furthermore, CAZ-3 reduced the tumour levels to $34 \%$ of control untreated tumour size. This value is below the $\mathrm{T} / \mathrm{C}$ value of $42 \%$ which is defined as the minimum level of activity required by the National Cancer Institute criteria. However, despite an excellent tumour response rate, a single i.p. injection of CAZ-3 at $40 \mathrm{mg} / \mathrm{kg}$ gave adverse side effects such as rough coat, loss of appetite and diarrhoea along with a mortality rate of $1 / 5$. The single maximum tolerated dose for CA-4P was $360 \mathrm{mg} / \mathrm{m}^{2}$ in rats and $100 \mathrm{mg} / \mathrm{m}^{2}$ in dogs (22). Serious diarrhoea has been reported elsewhere in animals given an i.p. injection of CA-4 at $100 \mathrm{mg} / \mathrm{kg}(23)$. CA-4P (100 mg/kg) had a mortality rate of $25 \%$ in rats (http://arno.unimaas.nl/show.cgi?fid=7247). In $90 \%$ of patients CA-4P is well tolerated at $52 \mathrm{mg} / \mathrm{m}^{2}(22)$. Given that CAZ-3 is intrinsically more stable than CA-4 and has a slower intrinsic clearance time, CAZ-3 may be more active in vivo as well as in vitro and thus may require significantly lower dosing rates. Optimising the dosing schedule and/or administration route may yield more favourable results.

In conclusion, we have presented preclinical data on a novel series of $c i s$-stable combretastatin analogues. We demonstrate that our lead compound CAZ-3 is effective against CA-4 resistant colon cancer-derived cells in vitro and in vivo. Further studies are warranted to characterise the metabolites of CAZ-3 and optimise dosing schedules with a view to improving the therapeutic potential of this novel class of $c i s$-stable vascular targeting agents.

\section{Acknowledgements}

We would like to thank Health Research Board Ireland for funding the project. We would also like to thank Sally Lee at Cyprotex Discovery, Ltd., (Macclesfield, UK) for carrying out the microsomal stability studies.

\section{References}

1. Pettit GR, Singh SB, Hamel E, Lin CM, Alberts DS and GarciaKendall D: Isolation and structure of the strong cell growth and tubulin inhibitor combretastatin A-4. Experientia 45: 209-211, 1989.

2. Marrelli M, Conforti F, Statti GA, Cachet X, Michel S, Tillequin F and Menichini F: Biologicalpotential and structure-activity relationships of most recently developed vascular disrupting agents: an overview of new derivatives of natural combretastatin a-4. Curr Med Chem 18: 3035-3081, 2011. 
3. Dowlati A, Robertson K, Cooney M, Petros WP, Stratford M, Jesberger J, Rafie N, Overmoyer B, Makkar V, Stambler B, Taylor A, Waas J, Lewin JS, McCrae KR and Remick SC: A phase I pharmacokinetic and translational study of the novel vascular targeting agent combretastatin a- 4 phosphate on a single-dose intravenous schedule in patients with advanced cancer. Cancer Res 62: 3408-3416, 2002

4. Delmonte A and Sessa C: AVE8062: a new combretastatin derivative vascular disrupting agent. Expert Opin Investig Drugs 18: 1541-1548, 2009

5. Beale TM, Myers RM, Shearman JW, Charnock-Jones SD, Brenton JD, Gergely FV and Ley SV: Antivascular and anticancer activity of dihalogenated A-ring analogues of combretastatin A-4. Med Chem Commun 1: 202-208, 2010.

6. Shan Y, Zhang J, Liu Z, Wang M and Dong Y: Developments of combretastatin A-4 derivatives as anticancer agents. Curr Med Chem 18: 523-538, 2011.

7. O'Boyle NM, Greene LM, Bergin O, Fichet JB, McCabe T, Lloyd DG, Zisterer DM and Meegan MJ: Synthesis, evaluation and structural studies of antiproliferative tubulin-targeting azetidin-2-ones. Bioorg Med Chem 19: 2306-2325, 2011.

8. Carr M, Greene LM, Knox AJ, Lloyd DG, Zisterer DM and Meegan MJ: Lead identification of conformationally restricted beta-lactam type combretastatin analogues: synthesis, antiproliferative activity and tubulin targeting effects. Eur J Med Chem 45: 5752-5766, 2010

9. O'Boyle NM, Carr M, Greene LM, Bergin O, Nathwani SM, McCabe T, Lloyd DG, Zisterer DM and Meegan MJ: Synthesis and evaluation of azetidinone analogues of combretastatin A-4 as tubulin targeting agents. J Med Chem 53: 8569-8584, 2010.

10. Greene LM, O'Boyle NM, Nolan DP, Meegan MJ and Zisterer DM: The vascular targeting agent Combretastatin-A4 directly induces autophagy in adenocarcinoma-derived colon cancer cells. Biochem Pharmacol 84: 612-624, 2012.

11. O'Boyle NM, Carr M, Greene LM, Keely NO, Knox AJ, McCabe T, Lloyd DG, Zisterer DM and Meegan MJ: Synthesis, biochemical and molecular modelling studies of antiproliferative azetidinones causing microtubule disruption and mitotic catastrophe. Eur J Med Chem 46: 4595-4607, 2011.

12. Schobert R, Effenberger-Neidnicht K and Biersack B: Stable combretastatin A-4 analogues with sub-nanomolar efficacy against chemoresistant HT-29 cells. Int J Clin Pharmacol Ther 49: 71-72, 2011.

13. Ohsumi K, Nakagawa R, Fukuda Y, Hatanaka T, Morinaga Y Nihei Y, Ohishi K, Suga Y, Akiyama Y and Tsuji T: Novel combretastatin analogues effective against murine solid tumors: design and structure-activity relationships. J Med Chem 41: 3022-3032, 1998.
14. Hinnen $\mathrm{P}$ and Eskens FA: Vascular disrupting agents in clinical development. Br J Cancer 96: 1159-1165, 2007

15. Patterson DM, Zweifel M, Middleton MR, Price PM, Folke LK, Stratford MR, Ross P, Halford S, Peters J, Balkissoon J, Chaplin DJ, Padhani AR and Rustin GJ: Phase I clinical and pharmacokinetic evaluation of the vascular-disrupting agent OXi4503 in patients with advanced solid tumors. Clin Cancer Res 18: 1415-25, 2012.

16. Greene LM, Nathwani SM, Bright SA, Fayne D, Croke A, Gagliardi M, McElligott AM, O'Connor L, Carr M, Keely NO, O'Boyle NM, Carroll P, Sarkadi B, Conneally E, Lloyd DG, Lawler M, Meegan MJ and Zisterer DM: The vascular targeting agent combretastatin-A4 and a novel cis-restricted $\beta$-lactam analogue, CA-432, induce apoptosis in human chronic myeloid leukemia cells and ex vivo patient samples including those displaying multidrug resistance. J Pharmacol Exp Ther 335: 302-313, 2010.

17. Greene LM, Carr M, Keeley NO, Lawler M, Meegan MJ and Zisterer DM: BubR1 is required for the mitotic block induced by combretastatin-A4 and a novel cis-restricted $\beta$-lactam analogue in human cancer cells. Int J Mol Med 27: 715-723, 2011.

18. Rubinsztein DC, Codogno P and Levine B: Autophagy modulation as a potential therapeutic target for diverse diseases. Nat Rev Drug Discov 11: 709-730, 2012.

19. Leslie EM, Deeley RG and Cole SP: Toxicological relevance of the multidrug resistance protein 1, MRP1 (ABCC1) and related transporters. Toxicology 167: 3-23, 2001.

20. Lee HY, Chang JY, Nien CY, Kuo CC, Shih KH, Wu CH, Chang CY, Lai WY and Liou JP: 5-Amino-2-aroylquinolines as highly potent tubulin polymerization inhibitors. Part 2 . The impact of bridging groups at position C-2. J Med Chem 54: 8517-8525, 2011.

21. Romagnoli R, Baraldi PG, Salvador MK, Preti D, Aghazadeh Tabrizi M, Brancale A, Fu XH, Li J, Zhang SZ, Hamel E, Bortolozzi R, Basso G and Viola G: Synthesis and evaluation of 1,5-disubstituted tetrazoles as rigid analogues of combretastatin A-4 with potent antiproliferative and antitumor activity. J Med Chem 55: 475-488, 2012.

22. Rustin GJ, Galbraith SM, Anderson H, Stratford M, Folkes LK, Sena L, Gumbrell L and Price PM: Phase I clinical trial of weekly combretastatin A4 phosphate: clinical and pharmacokinetic results. J Clin Oncol 21: 2815-2822, 2003.

23. Eikesdal HP, Schem BC, Mella O and Dahl O: The new tubulininhibitor combretastatin A-4 enhances thermal damage in the BT4An rat glioma. Int J Radiat Oncol Biol Phys 46: 645-652, 2000 . 